\title{
A reverse quasiconformal composition problem for $Q_{\alpha}\left(\mathbb{R}^{n}\right)$
}

\author{
Jie Xiao and Yuan Zhou
}

\begin{abstract}
We give a partial converse to [8, Theorem 1.3] (as a resolution of [2, Problem 8.4] for the quasiconformal Q-composition) for $Q_{0<\alpha<2^{-1}}\left(\mathbb{R}^{n \geq 2}\right)$, and yet demonstrate that if $f: \mathbb{R}^{2} \rightarrow \mathbb{R}^{2}$ is a homeomorphism then the boundedness of $u \mapsto u \circ f$ on $Q_{2-1<\alpha<1}\left(\mathbb{R}^{2}\right) \subset B M O\left(\mathbb{R}^{2}\right)$ yields the quasiconformality of $f$.
\end{abstract}

\section{Introduction}

Recall that $Q_{-\infty<\alpha<\infty}\left(\mathbb{R}^{n}\right)$ is the quite-well-known Essén-Janson-Peng-Xiao's space of all measurable functions $u$ on $\mathbb{R}^{n \geq 1}$ with

$$
\|u\|_{Q_{\alpha}\left(\mathbb{R}^{n}\right)}=\sup _{\left(x_{0}, r\right) \in \mathbb{R}^{n} \times(0, \infty)}\left(r^{2 \alpha-n} \int_{\left|y-x_{0}\right|<r} \int_{\left|x-x_{0}\right|<r} \frac{|u(x)-u(y)|^{2}}{|x-y|^{n+2 \alpha}} d x d y\right)^{\frac{1}{2}}<\infty .
$$

In particular (cf. [2], [5]),

$$
Q_{0 \leq \alpha<\infty}\left(\mathbb{R}^{n}\right) \subset Q_{-\infty<\alpha<0}\left(\mathbb{R}^{n}\right)=Q_{-\frac{n}{2}}\left(\mathbb{R}^{n}\right)=B M O\left(\mathbb{R}^{n}\right) .
$$

As a resolution of $\left[2\right.$, Problem 8.4] - Let $f$ be a quasiconformal self-map of $\mathbb{R}^{n}$. Prove or disprove that $u \mapsto \mathbf{C}_{f} u=u \circ f$ is bounded on $Q_{0<\alpha<1}\left(\mathbb{R}^{n \geq 2}\right.$ ) (which however has an affirmative solution for $B M O\left(\mathbb{R}^{n}\right)$ as proved in $[9$, Theorem 2] - namely $\mathbf{C}_{f}$ is bounded on $B M O\left(\mathbb{R}^{n}\right)$ whenever $f$ is a quasiconformal self-map of $\left.\mathbb{R}^{n}\right)$, we have

JX is supported by NSERC of Canada (\# 202979463102000); YZ is supported by AvHfoundation and National Natural Science Foundation of China (\# 11522102 \& 11871088) respectively.

$\mathrm{YZ}$ is corresponding author. reverse.

Key words and phrases: quasiconformality, composition, Essén-Janson-Peng-Xiao's space, 2010 Mathematics Subject Classification: 42B35, 46E30, 47B38, 30H25. 
Theorem 1.1. [8, Theorem 1.3] For $n-1 \in \mathbb{N}$ let $f: \mathbb{R}^{n} \rightarrow \mathbb{R}^{n}$ be quasiconformal. If there exists a closed set $E \subseteq \mathbb{R}^{n}$ such that

$\triangleright J_{f}$, the Jacobian determinant of $f$, belongs to the E-based Muckenhoupt class $A_{1}\left(\mathbb{R}^{n} ; E\right)$;

$\triangleright \overline{\operatorname{dim}}_{L} E$ (under $E$ being bounded) or $\overline{\operatorname{dim}}_{L G} E$ (under $E$ being unbounded), the local or global self-similar Minkowski dimension of $E$ (bounded or unbounded), lies in $[0, n-2]$, i.e.,

$$
[0, n-2] \ni \begin{cases}\overline{\operatorname{dim}}_{L} E & \text { as } E \text { is bounded; } \\ \overline{\operatorname{dim}}_{L G} E & \text { as } E \text { is unbounded, }\end{cases}
$$

then $\mathbf{C}_{f}$ is bounded on $Q_{0<\alpha<1}\left(\mathbb{R}^{n}\right)$.

As a partial converse to Theorem 1.1, we here show

Theorem 1.2. For $n-1 \in \mathbb{N}$ let $f: \mathbb{R}^{n} \rightarrow \mathbb{R}^{n}$ be a homeomorphism. If

$\triangleright \mathbf{C}_{f}$ and $\mathbf{C}_{f^{-1}}$ are bijective and bounded on $Q_{0<\alpha<2^{-1}}\left(\mathbb{R}^{n}\right)$ respectively;

$\triangleright f$ is not only ACL (absolutely continuous on almost all lines parallel to coordinates of $\mathbb{R}^{n}$ ) but also differentiable almost everywhere on $\mathbb{R}^{n}$, then $f$ is quasiconformal.

Remark 1.3. Below are two comments on Theorem 1.2.

(i) Under the above assumptions on $f$, we have that $f^{-1}$ is absolutely continuous with respect to the $n$-dimensional Lebesgue measure. Indeed, let $f^{-1}$ map a set $N$ of the $n$-dimensional Lebesgue measure 0 to a set $O=f^{-1}(N)$. If $\chi_{N}$ and $\chi_{O}$ stand for the indicators of $N$ and $O$ respectively, then $k \chi_{O}, k \chi_{N} \in Q_{0<\alpha<2^{-1}}\left(\mathbb{R}^{n}\right)$ for any $k \in \mathbb{N}$, but $k \chi_{N}=0$ in $Q_{0<\alpha<2^{-1}}\left(\mathbb{R}^{n}\right)$, and hence from the first $\triangleright$-hypothesis in Theorem 1.2 it follows that $k \chi_{O}=0$ in $Q_{0<\alpha<2^{-1}}\left(\mathbb{R}^{n}\right)$ and so $O=f^{-1}(N)$ is of the $n$-dimensional Lebesgue measure 0 .

(ii) In accordance with [9, Theorem 3] (cf. [1, Theorem] \& [3, Theorem 3.1] for some generalizations), we have that if the first requirement on $\mathbf{C}_{f} \& \mathbf{C}_{f^{-1}}$ in Theorem 1.2 is replaced by the condition that $f^{-1}$ is absolutely continuous and the second requirement on $f$ is kept the same then the boundedness of $\mathbf{C}_{f}$ on $B M O\left(\mathbb{R}^{n}\right)$ derives that $f$ is a quasiconformal self-map of $\mathbb{R}^{n}$. Accordingly, this $B M O\left(\mathbb{R}^{n}\right)$-result can be naturally strengthened via Theorem 1.2 thanks to $Q_{0<\alpha<2^{-1}}\left(\mathbb{R}^{n}\right) \subset B M O\left(\mathbb{R}^{n}\right)$.

In addition, while focusing on the planar situation of Theorem 1.1 and observing that the Jacobian determinant of any quasiconformal self-map of $\mathbb{R}^{n \geq 2}$ is an $A_{\infty}$-weight (cf. [4, Theorem 15.32]) we readily discover

Theorem 1.4. [8, Theorem 1.3: $n=2 \& E=\varnothing]$ Let $f: \mathbb{R}^{2} \rightarrow \mathbb{R}^{2}$ be quasiconformal. If $J_{f}$ is an $A_{1}$-weight on $\mathbb{R}^{2}$, i.e., $J_{f} \in A_{1}\left(\mathbb{R}^{2} ; \varnothing\right)$, then $\mathbf{C}_{f}$ is bounded on $Q_{0<\alpha<1}\left(\mathbb{R}^{2}\right)$. 
On the basis of the planar cases of Theorem 1.2 and Remark 1.3(ii), a partial converse to Theorem 1.4 (under $2^{-1}<\alpha<1$ ) is naturally given by

Theorem 1.5. Let $f: \mathbb{R}^{2} \rightarrow \mathbb{R}^{2}$ be a homeomorphism. If $\mathbf{C}_{f}$ is bounded on $Q_{2^{-1}<\alpha<1}\left(\mathbb{R}^{2}\right)$, then $f$ is quasiconformal.

Remark 1.6. Let $n \geq 2$. Recall that if a homeomorphism of $\mathbb{R}^{n}$ preserves either the Sobolev space $W^{1, n}\left(\mathbb{R}^{n}\right)$ or the Triebel-Lizorkin space $\dot{F}_{n / s, q}^{s}\left(\mathbb{R}^{n}\right)$ with $s \in$ $(0,1) \& q \in[1, \infty)$, it must be quasiconformal. But any homeomorphism preserving the Besov space $\dot{B}_{n / s, q}^{s}\left(\mathbb{R}^{n}\right)$ with $s \in(0,1) \& q \in[1, \infty) \backslash\{n / s\}$ or $s \in(0,1) \& q=n / s$ must be bi-Lipschitz or quasiconformal; see also [6], [7] and the references therein. By Reimann's paper [9], a homeomorphism of $\mathbb{R}^{n}$ preserving the John-Nirenberg space $B M O\left(\mathbb{R}^{n}\right)$ and satisfying the assumptions of Theorem 1.2 must be quasiconformal.

The rest of this paper is organized as follows: $\S 2$ is employed to prove Theorem 1.2 in terms of Lemmas 2.1-2.2 \& $2.4 \& 2.6$ as well as Corollaries $2.3 \& 2.5$ producing a suitable $Q_{\alpha}\left(\mathbb{R}^{n}\right)$-function. More precisely, we borrow some of Reimann's ideas from [9] to prove Theorem 1.2, namely, prove that

$$
\sup _{y \in \mathbb{R}^{n} \&|y|=1}\left|\left(D f^{-1}(x)\right) y\right|^{n} \lesssim J_{f^{-1}}(x)
$$

holds for almost all $x \in \mathbb{R}^{n}$, where $D f^{-1}$ and $J_{f^{-1}}$ are the formal derivative and Jacobian determinant of $f^{-1}$ (cf. [4, Chapters 14-15]) - equivalently - we show that the maximal eigenvalue $\lambda_{1}$ of $D f^{-1}(x)$ is bounded by the minimal eigenvalue $\lambda_{n}$ of $D f^{-1}(x)$ - in fact - by comparing the norms of suitable scalings of some special $Q_{\alpha}\left(\mathbb{R}^{n}\right)$-functions $u_{\star}$ (cf. Corollary $2.5 \&$ Lemma 2.6) and their compositions with $f$, we can obtain the desired inequality $\lambda_{1} \lesssim \lambda_{n}$. 33 is designed to demonstrate Theorem 1.5 through a $Q_{\alpha}\left(\mathbb{R}^{n}\right)$-capacity estimate given in Lemma 3.1 and a technique for reducing the space dimension shown in Lemma 2.1.

Notation In the above and below, $X \lesssim Y$ stands for $X \leq \varkappa Y$ with a constant $\varkappa>0$.

\section{Validation of Theorem 1.2}

In order to prove the validity of Theorem 1.2, we need four lemmas and two corollaries.

Lemma 2.1. Let $(\alpha, n, m) \in \mathbb{R} \times \mathbb{N} \times \mathbb{N}$ and $u: \mathbb{R}^{n} \rightarrow \mathbb{R}$. Then $u \in Q_{\alpha}\left(\mathbb{R}^{n}\right)$ if and only if $\mathbb{R}^{n} \times \mathbb{R}^{m} \ni(x, y) \mapsto U(x, y)=u(x)$ belongs to $Q_{\alpha}\left(\mathbb{R}^{n+m}\right)$. 
Proof. This follows immediately from [2, Theorem 2.6] and its demonstration.

Lemma 2.2. Let $(\alpha, n) \in\left[0, \min \left\{1,2^{-1} n\right\}\right) \times \mathbb{N}$. Then $x \mapsto \ln |x|$ is in $Q_{\alpha}\left(\mathbb{R}^{n}\right)$.

Proof. For any Euclidean ball $B=B\left(x_{0}, r\right)$ with centre $x_{0} \in \mathbb{R}^{n}$ and radius $r \in$ $(0, \infty)$ and a measurable function $u$ on $\mathbb{R}^{n}$ let

$$
\Phi_{\alpha}(u, B)=r^{2 \alpha-n} \int_{B} \int_{B} \frac{|u(x)-u(y)|^{2}}{|x-y|^{n+2 \alpha}} d x d y
$$

So, it suffices to verify that if $u_{\ln }(x)=\ln |x|$ then $\Phi_{\alpha}\left(u_{\ln }, B\right) \lesssim 1$.

- Case $\left|x_{0}\right|>2 r$. Note that there is $\theta \in(0,1)$ obeying

$$
\begin{aligned}
x, y \in B & \Longrightarrow r<|x|,|y| \leq 3 r \\
& \Longrightarrow|\ln | x|-\ln | y||=\frac{|| x|-| y||}{(1-\theta)|x|+\theta|y|} \leq \frac{|x-y|}{r} .
\end{aligned}
$$

So

$$
\begin{aligned}
\Phi_{\alpha}\left(u_{\ln }, B\right) & =r^{2 \alpha-n-2} \int_{B} \int_{B}|x-y|^{2-n-2 \alpha} d x d y \\
& \leq r^{2 \alpha-n-2} \int_{B} \int_{B(x, 2 r)}|x-y|^{2-n-2 \alpha} d y d x \\
& \lesssim r^{2 \alpha-2} \int_{0}^{r} t^{1-2 \alpha} d t \\
& \lesssim 1
\end{aligned}
$$

as desired.

- Case $\left|x_{0}\right| \leq 2 r$. Since $B\left(x_{0}, r\right) \subseteq B(0,3 r)$ - the origin-centered ball with radius $3 r$, we only need to estimate $\Phi_{\alpha}\left(u_{\ln }, B\right)$ for $B=B(0, r)$.

Firstly, write

$$
\left\{\begin{array}{l}
\Phi_{\alpha}\left(u_{\ln }, B\right)=I_{1}+I_{2}+I_{3} \\
I_{1}=r^{2 \alpha-n} \int_{B} \int_{B\left(x, 2^{-1}|x|\right)} \frac{\left.|\ln | x|-\ln | y\right|^{2}}{|x-y|^{n+2 \alpha}} d y d x \\
I_{2}=r^{2 \alpha-n} \int_{B} \int_{B \backslash B(x, 4|x|)} \frac{\left.|\ln | x|-\ln | y\right|^{2}}{|x-y|^{n+2 \alpha}} d y d x \\
I_{3}=r^{2 \alpha-n} \int_{B} \int_{B(x, 4|x|) \backslash B\left(x, 2^{-1}|x|\right)} \frac{|\ln | x|-\ln | y||^{2}}{|x-y|^{n+2 \alpha}} d y d x .
\end{array}\right.
$$

Since

$$
|x-y| \leq 2^{-1}|x| \Longrightarrow|\ln | x|-\ln | y|| \leq 2|x-y||x|^{-1},
$$

one has

$$
I_{1} \lesssim r^{2 \alpha-n} \int_{B}|x|^{-2} \int_{B\left(x, 2^{-1}|x|\right)}|x-y|^{2-n-2 \alpha} d y d x \lesssim 1
$$


Secondly, write

$$
\int_{B \backslash B(x, 4|x|)} \frac{|\ln | x|-\ln | y||^{2}}{|x-y|^{n+2 \alpha}} d y \leq \sum_{j \geq 3} \int_{B\left(x, 2^{j}|x|\right) \backslash B\left(x, 2^{j-1}|x|\right)} \frac{|\ln | x|-\ln | y||^{2}}{|x-y|^{n+2 \alpha}} d y .
$$

Observe that if $j-2 \in \mathbb{N}$ then

$$
\begin{aligned}
2^{j-1}|x| \leq|x-y| \leq 2^{j}|x| & \Longrightarrow 2^{j-2}|x| \leq|y| \leq 2^{j+1}|x| \\
& \Longrightarrow \int_{B\left(x, 2^{j}|x|\right) \backslash B\left(x, 2^{j-1}|x|\right)} \frac{\left.|\ln | x|-\ln | y\right|^{2}}{|x-y|^{n+2 \alpha}} d y \lesssim \frac{2^{j(2-2 \alpha)}}{|x|^{2 \alpha}} .
\end{aligned}
$$

Thus

$$
I_{2} \lesssim r^{2 \alpha-n} \int_{B}|x|^{-2 \alpha} \sum_{j=3}^{\infty}(\ldots) d y \lesssim r^{2 \alpha-n} \int_{B}|x|^{-2 \alpha} d x \lesssim 1
$$

Thirdly, note that

$$
y \in B(x, 4|x|) \backslash B\left(x, 2^{-1}|x|\right) \Longrightarrow|y| \leq 5|x| .
$$

So

$$
\begin{aligned}
I_{3} & \lesssim r^{2 \alpha-n} \int_{B} \int_{B(x, 4|x|) \backslash B\left(x, 2^{-1}|x|\right)} \frac{\left.|\ln | x|-\ln | y\right|^{2}}{|x-y|^{n+2 \alpha}} d y d x \\
& \lesssim r^{2 \alpha-n} \int_{B}|x|^{-(n+2 \alpha)} \int_{B(0,5|x|)}\left(\ln \frac{|x|}{|y|}\right)^{2} d y d x \\
& \lesssim r^{2 \alpha-n} \int_{B}|x|^{-(n+2 \alpha)} \sum_{i=1}^{\infty}\left(2^{-i} 5|x|\right)^{n} i^{2} d x \\
& \lesssim r^{2 \alpha-n} \int_{B}|x|^{-2 \alpha} d x \\
& \lesssim 1 .
\end{aligned}
$$

Corollary 2.3. Let $(n-1, c) \in \mathbb{N} \times \mathbb{R}$. Then

(i)

$$
x=\left(x_{1}, x_{2}, \ldots, x_{n}\right) \longmapsto \max \left\{c, \ln \left(x_{1}^{-2}\right)\right\}
$$

is in $Q_{0 \leq \alpha<2^{-1}}\left(\mathbb{R}^{n}\right)$.

(ii)

$$
x=\left(x_{1}, x_{2}, \ldots, x_{n}\right) \longmapsto \max \left\{c, \ln \left(x_{1}^{2}+x_{2}^{2}\right)^{-1}\right\}
$$

is in $Q_{0 \leq \alpha<1}\left(\mathbb{R}^{n}\right)$. 
Proof. This follows from

$$
\max \{u, v\}=2^{-1}(u+v+|u-v|)=u+\max \{v-u, 0\},
$$

the basic fact that $Q_{\alpha}\left(\mathbb{R}^{n}\right)$ is a linear space with

$$
w \in Q_{\alpha}\left(\mathbb{R}^{n}\right) \Longrightarrow|w| \in Q_{\alpha}\left(\mathbb{R}^{n}\right),
$$

and Lemmas 2.1-2.2.

Lemma 2.4. Let $(\alpha, n-1) \in(0,1) \times \mathbb{N}$. If

$$
\left\{\begin{array}{l}
\left|\|u \mid\|_{Q_{\alpha}}=\|u\|_{Q_{\alpha}\left(\mathbb{R}^{n}\right)}+\sup _{\left(x_{0}, r\right) \in \mathbb{R}^{n} \times[1, \infty)}\left(r^{2 \alpha-n} \int_{B\left(x_{0}, r\right)}|u(x)|^{2} d x\right)^{2^{-1}}<\infty ;\right. \\
\|g\|_{\infty, L i p}=\|g\|_{L^{\infty}(\mathbb{R})}+\sup _{z_{1}, z_{2} \in \mathbb{R}, z_{1} \neq z_{2}}\left|g\left(z_{1}\right)-g\left(z_{2}\right) \| z_{1}-z_{2}\right|^{-1}<\infty,
\end{array}\right.
$$

then $\mathbb{R}^{n} \times \mathbb{R} \ni(x, z) \mapsto u(x) g(z)$ belongs to $Q_{\alpha}\left(\mathbb{R}^{n} \times \mathbb{R}\right)$.

Proof. For any

$$
\left(x_{0}, z_{0}, \rho, r, k+2\right) \in \mathbb{R}^{n} \times \mathbb{R} \times(0, \infty) \times(0, \infty) \times \mathbb{N},
$$

set

$$
\left\{\begin{array}{l}
C\left(x_{0}, z_{0}, \rho\right)=\left\{(x, z) \in \mathbb{R}^{n} \times \mathbb{R}:\left|\left(x-x_{0}, z-z_{0}\right)\right| \leq \rho\right\} \\
A\left(k, x_{0}, z_{0}, r\right)=C\left(x_{0}, z_{0}, 2^{-k} r\right) \backslash C\left(x_{0}, z_{0}, 2^{-k-1} r\right) \\
a_{k, r}\left(x_{0}, z_{0}\right)=u_{A\left(k, x_{0}, z_{0}, r\right)} g\left(z_{0}\right)
\end{array}\right.
$$

Here and henceforth, for a given set $E \subset \mathbb{R}^{m \geq 1}$ with the $m$-dimensional Lebesgue measure $|E|>0$, the symbol

$$
u_{E}=f_{E} u(x) d x=|E|^{-1} \int_{E} u(x) d x
$$

stands for the average of $u$ over $E$. We make the following claim

$$
\begin{aligned}
\Psi_{\alpha} & \left(u g, C\left(x_{0}, z_{0}, r\right)\right) \\
& :=\sum_{k \geq-1} 2^{2 k \alpha} f_{C\left(x_{0}, z_{0}, r\right)} f_{A(k, x, z, r)}\left|u(\tilde{x}) g(\tilde{z})-a_{k, r}(x, z)\right|^{2} d \tilde{z} d \tilde{x} d z d x \\
& \lesssim\left(\|g\|_{\infty, L i p}\left|\|u \mid\|_{Q_{\alpha}}\right)^{2} .\right.
\end{aligned}
$$

Assume that the last estimation holds for the moment. Then an application of the basic fact that

$$
\left\{\begin{array}{l}
C(x, z, 2 r)=\bigcup_{k \geq-1} A(k, x, z, r) \\
A(k, x, z, r) \cap A(l, x, z, r)=\varnothing \quad \forall k \neq l ; \\
((x, z),(y, w)) \in C\left(x_{0}, z_{0}, r\right) \times C\left(x_{0}, z_{0}, r\right) \Longrightarrow(y, w) \in C(x, z, 2 r) \subset C\left(x_{0}, z_{0}, 3 r\right),
\end{array}\right.
$$


the Hölder inequality and Lemma 2.1 gives

$$
\begin{aligned}
& r^{2 \alpha-n-1} \int_{C\left(x_{0}, z_{0}, r\right)} \int_{C\left(x_{0}, z_{0}, r\right)} \frac{|u(x) g(z)-u(y) g(w)|^{2}}{|(x, z)-(y, w)|^{n+1+2 \alpha}} d x d z d y d w \\
& \lesssim r^{2 \alpha} f_{C\left(x_{0}, z_{0}, r\right)} \int_{C(x, z, 2 r)} \frac{|u(x) g(z)-u(y) g(w)|^{2}}{|(x, z)-(y, w)|^{n+1+2 \alpha}} d y d w d x d z \\
& \lesssim f_{C\left(x_{0}, z_{0}, r\right)} \sum_{k \geq-1} \frac{2^{2 k \alpha}}{\left(2^{-k} r\right)^{n+1}} \int_{A(k, x, z, r)} \frac{d y d w d x d z}{|u(x) g(z)-u(y) g(w)|^{-2}} \\
& \lesssim f_{C\left(x_{0}, z_{0}, r\right)} \sum_{k \geq-1} f_{A(k, x, z, r)} \frac{2^{2 k \alpha} d y d w d x d z}{\left|\left(a_{k, r}(x, z)-u(y) g(w)\right)+\left(u(x) g(z)-a_{k, r}(x, z)\right)\right|^{-2}} \\
& \lesssim \Psi_{\alpha}\left(u g, C\left(x_{0}, z_{0}, r\right)\right)+\|g\|_{\infty, L i p}^{2} \sum_{k \geq-1} 2^{2 k \alpha} f_{C\left(x_{0}, z_{0}, r\right)}\left|u(x)-u_{A(k, x, z, r)}\right|^{2} d x d z \\
& \lesssim \Psi_{\alpha}\left(u g, C\left(x_{0}, z_{0}, r\right)\right) \\
& +\|g\|_{\infty, L i p}^{2} \sum_{k \geq-1} 2^{2 k \alpha} f_{C\left(x_{0}, z_{0}, r\right)} f_{A(k, x, z, r)}|u(x)-u(y)|^{2} d y d w d x d z \\
& \lesssim \Psi_{\alpha}\left(u g, C\left(x_{0}, z_{0}, r\right)\right) \\
& +\|g\|_{\infty, L i p}^{2} \sum_{k \geq-1} \int_{C\left(x_{0}, z_{0}, r\right)} \int_{A(k, x, z, r)} \frac{|u(x)-u(y)|^{2} d y d w d x d z}{r^{n+1-2 \alpha}|(x-y, z-w)|^{1+n+2 \alpha}} \\
& \lesssim \Psi_{\alpha}\left(u g, C\left(x_{0}, z_{0}, r\right)\right) \\
& +\|g\|_{\infty, L i p}^{2} \int_{C\left(x_{0}, z_{0}, 3 r\right)} \int_{C(x, z, 2 r) \subset C\left(x_{0}, z_{0}, 3 r\right)} \frac{|u(x)-u(y)|^{2} d y d w d x d z}{r^{n+1-2 \alpha}|(x-y, z-w)|^{1+n+2 \alpha}} \\
& \lesssim \Psi_{\alpha}\left(u g, C\left(x_{0}, z_{0}, r\right)\right)+\left(\|g\|_{\infty, L i p}\left|\|u \mid\|_{Q_{\alpha}}\right)^{2} .\right.
\end{aligned}
$$

This, plus the foregoing claim, yields

$$
\begin{aligned}
& \|u g\|_{Q_{\alpha}\left(\mathbb{R}^{n+1}\right)}^{2} \\
& \quad=\sup _{\left(x_{0}, z_{0}, r\right) \in \mathbb{R}^{n} \times \mathbb{R} \times(0, \infty)} \int_{C\left(x_{0}, z_{0}, r\right)} \int_{C\left(x_{0}, z_{0}, r\right)} \frac{|u(x) g(z)-u(y) g(w)|^{2}}{|(x, z)-(y, w)|^{n+1+2 \alpha}} \frac{d x d z d y d w}{r^{n+1-2 \alpha}} \\
& \quad \lesssim \sup _{\left(x_{0}, z_{0}, r\right) \in \mathbb{R}^{n} \times \mathbb{R} \times(0, \infty)} \Psi_{\alpha}\left(u g, C\left(x_{0}, z_{0}, r\right)\right)+\left(\|g\|_{\infty, L i p}\left|\|u \mid\|_{Q_{\alpha}}\right)^{2}\right. \\
& \quad \lesssim\left(\|g\|_{\infty, L i p}\left|\|u \mid\|_{Q_{\alpha}}\right)^{2},\right.
\end{aligned}
$$

Now, it remains to verify the above claim.

First of all, we have

$f_{A(k, x, x, r)}\left|u(\tilde{x}) g(\tilde{z})-a_{k, r}(x, z)\right|^{2} d \tilde{x} d \tilde{z}$ 


$$
\begin{aligned}
& \lesssim f_{A(k, x, z, r)}\left|u(\tilde{x})-u_{A(k, x, z, r)}\right|^{2}|g(\tilde{z})|^{2} d \tilde{x} d \tilde{z}+f_{A(k, x, z, r)} \frac{|g(\tilde{z})-g(z)|^{2}}{\left|u_{A(k, x, z, r)}\right|^{-2}} d \tilde{x} d \tilde{z} \\
& \lesssim\|g\|_{\infty, L i p}^{2}\left(f_{A(k, x, z, r)}\left|u(\tilde{x})-u_{A(k, x, z, r)}\right|^{2} d \tilde{x} d \tilde{z}+\min \left\{2^{-k} r, 1\right\}^{2}\left|u_{A(k, x, z, r)}\right|^{2}\right)
\end{aligned}
$$

thereby finding that if

$$
I(u, \alpha)=\sum_{k \geq-1} 2^{2 k \alpha} \min \left\{2^{-k} r, 1\right\}^{2} f_{C\left(x_{0}, z_{0}, r\right)}\left|u_{A(k, x, z, r)}\right|^{2} d x d z
$$

then an application of the triangle inequality, the Hölder inequality and Lemma 2.1 derives

$$
\begin{aligned}
\Psi_{\alpha}\left(u g, C\left(x_{0}, z_{0}, r\right)\right) & \\
\lesssim & \|g\|_{\infty, L i p}^{2}\left(\sum_{k \geq-1} 2^{2 k \alpha} f_{C\left(x_{0}, z_{0}, r\right)} f_{A(k, x, z, r)}\left|u(\tilde{x})-u_{A(k, x, z, r)}\right|^{2} d \tilde{x} d \tilde{z} d x d z\right. \\
& +I(u, \alpha)) \\
\lesssim & \|g\|_{\infty, L i p}^{2}\left(\sum_{k \geq-1} 2^{2 k \alpha} f_{C\left(x_{0}, z_{0}, r\right)} f_{A(k, x, z, r)} \frac{d \tilde{x} d \tilde{z} d x d z}{\left(\left|u(x)-u_{A(k, x, z, r)}\right|^{2}+|u(\tilde{x})-u(x)|^{2}\right)^{-1}}\right. \\
& +I(u, \alpha)) \\
\lesssim & \|g\|_{\infty, L i p}^{2}\left(\left|\|u \mid\|_{Q_{\alpha}}^{2}\right.\right. \\
& +f_{C\left(x_{0}, z_{0}, r\right)} \sum_{k \geq-1} f_{A(k, x, z, r)} \frac{2^{2 k \alpha}|u(\tilde{x})-u(x)|^{2} d \tilde{x} d \tilde{z} d x d z}{|(\tilde{x}, \tilde{z})-(x, z)|^{1+n+2 \alpha}\left(2^{-k} r\right)^{-n-1-2 \alpha}} \\
& +I(u, \alpha)) \\
\lesssim & \|g\|_{\infty, L i p}^{2}\left(\left|\|u \mid\|_{Q_{\alpha}}^{2}\right.\right. \\
& +\int_{C\left(x_{0}, z_{0}, r\right)} \sum_{k \geq-1} \int_{A(k, x, z, r)} \frac{|u(\tilde{x})-u(x)|^{2} d \tilde{x} d \tilde{z} d x d z}{\left.|(\tilde{x})-(x, z)|^{1+n+2 \alpha} r^{1+n-2 \alpha}+I(u, \alpha)\right)} \\
\lesssim & \|g\|_{\infty, L i p}^{2}\left(\left|\|u \mid\|_{Q_{\alpha}}^{2}\right.\right.
\end{aligned}
$$




$$
\begin{aligned}
& \left.+\int_{C\left(x_{0}, z_{0}, 3 r\right)} \int_{C(x, z, 2 r) \subset C\left(x_{0}, z_{0}, 3 r\right)} \frac{|u(\tilde{x})-u(x)|^{2} d \tilde{x} d \tilde{z} d x d z}{|(\tilde{x}, \tilde{z})-(x, z)|^{1+n+2 \alpha} r^{1+n-2 \alpha}}+I(u, \alpha)\right) \\
& \lesssim\|g\|_{\infty, L i p}^{2}\left(\left|\|u \mid\|_{Q_{\alpha}}^{2}+I(u, \alpha)\right) .\right.
\end{aligned}
$$

Next, we handle $I(u, \alpha)$ according to the following two cases.

- Case $r<2$. By the hypothesis on $u$ and the inclusion

$$
Q_{\alpha}\left(\mathbb{R}^{n}\right) \subseteq B M O\left(\mathbb{R}^{n}\right)
$$

we obtain that if $k+2 \in \mathbb{N}$ then Lemma 2.1 yields

$$
\begin{aligned}
\left|u_{A(k, x, z, r)}\right| \lesssim\left(2^{-k} r\right)^{-n-1}\left|\int_{C\left(x, z, 2^{-k} r\right)} u(y) d y d w-\int_{C\left(x, z, 2^{-k-1} r\right)} u(y) d y d w\right| \\
\lesssim\left|u_{C\left(x, z, 2^{-k} r\right)}\right|+\left|u_{C\left(x, z, 2^{-k-1} r\right)}\right| \\
\lesssim\left|u_{C(x, z, 2)}\right|+\left|u_{C(x, z, 2)}-u_{C\left(x, z, 2^{-k} r\right)}\right|+\left|u_{C(x, z, 1)}\right| \\
\quad \quad+\left|u_{C(x, z, 1)}-u_{C\left(x, z, 2^{-k-1} r\right)}\right| \\
\lesssim\left(\left(|u|^{2}\right)_{B(x, 2)}\right)^{2^{-1}}+\left(\left(|u|^{2}\right)_{B(x, 1)}\right)^{2^{-1}}+\left(k+1+\ln \frac{4}{r}\right)\|u\|_{Q_{\alpha}\left(\mathbb{R}^{n}\right)} \\
\lesssim\left(k+2+\ln \frac{4}{r}\right)\left|\|u \mid\|_{Q_{\alpha}}\right.
\end{aligned}
$$

and hence

$$
I(u, \alpha) \lesssim\left|\left\|u \left|\left\|_ { Q _ { \alpha } } ^ { 2 } \sum _ { k \geq - 1 } 2 ^ { 2 k \alpha - 2 k } r ^ { 2 } ( k + 2 + \operatorname { l n } \frac { 4 } { r } ) ^ { 2 } \lesssim \left|\|u \mid\|_{Q_{\alpha}}^{2} .\right.\right.\right.\right.\right.
$$

- Case $r \geq 2$. An application of the hypothesis on $u$, the Hölder inequality and the Fubini theorem gives that if $k+2 \in \mathbb{N}$ then

$$
\begin{aligned}
& f_{C\left(x_{0}, z_{0}, r\right)}\left|u_{A(k, x, z, r)}\right|^{2} d x d z \\
& \quad \lesssim f_{C\left(x_{0}, z_{0}, r\right)}\left(|u|_{C\left(x, z, 2^{-k} r\right)}\right)^{2} d x d z \\
& \quad \lesssim f_{C\left(x_{0}, z_{0}, r\right)} f_{C\left(x, z, 2^{-k} r\right)}|u(y)|^{2} d y d w d x d z \\
& \quad \lesssim f_{C\left(x_{0}, z_{0}, r\right)} f_{C\left(0,0,2^{-k} r\right)}|u(x+z)|^{2} d x d z d y d w \\
& \quad \lesssim r^{-2 \alpha}\left|\|u \mid\|_{Q_{\alpha}}^{2}\right.
\end{aligned}
$$


and hence

$$
I(u, \alpha) \lesssim\left|\left\|u \left|\left\|_ { Q _ { \alpha } } ^ { 2 } ( \sum _ { k \geq \operatorname { l n } r } 2 ^ { 2 k \alpha - 2 k } r ^ { 2 - 2 \alpha } + \sum _ { - 1 \leq k \leq \operatorname { l n } r } 2 ^ { 2 k \alpha } r ^ { - 2 \alpha } ) \lesssim \left|\|u \mid\|_{Q_{\alpha}}^{2}\right.\right.\right.\right.\right.
$$

Finally, upon putting the previous two cases together, we achieve the desired estimation

$$
\Psi_{\alpha}\left(u g, C\left(x_{0}, z_{0}, r\right)\right) \lesssim\|g\|_{\infty, L i p}^{2}\left(| \| u | \| _ { Q _ { \alpha } } ^ { 2 } + I ( u , \alpha ) ) \lesssim \left(\|g\|_{\infty, L i p}\left|\|u \mid\|_{Q_{\alpha}}\right)^{2}\right.\right.
$$

Corollary 2.5. For $n-1 \in \mathbb{N}$ let

$$
\phi(t)= \begin{cases}0 & \text { as } t \in(-\infty,-2] \\ 1-|1+t| & \text { as } t \in[-2,0] \\ 1-|1-t| & \text { as } t \in[0,2] \\ 0 & \text { as } t \in[2, \infty)\end{cases}
$$

and

$$
\psi(t)= \begin{cases}1 & \text { as }|t| \leq 1 \\ 2-|t| & \text { as } 1 \leq|t| \leq 2 \\ 0 & \text { as }|t| \geq 2\end{cases}
$$

If

$$
u_{\star}\left(x_{1}, \ldots, x_{n}\right)= \begin{cases}\max \left\{0, \ln \left(x_{1}^{-2}\right)\right\} \phi\left(x_{2}\right) & \text { for } n=2 \\ \left(\max \left\{0, \ln \left(x_{1}^{-2}\right)\right\}\right) \psi\left(x_{2}\right) \ldots \psi\left(x_{n-1}\right) \phi\left(x_{n}\right) & \text { for } n \geq 3\end{cases}
$$

then $u_{\star} \in Q_{0<\alpha<2^{-1}}\left(\mathbb{R}^{n}\right)$.

Proof. Note that

$$
\|\phi\|_{\infty, L i p}+\|\psi\|_{\infty, L i p}<\infty
$$

holds and (via Corollary 2.3(i))

$$
u\left(x_{1}, \ldots, x_{n}\right)=\max \left\{0, \ln \left(x_{1}^{-2}\right)\right\} \quad \text { enjoys }\|u \mid\|_{Q_{0<\alpha<2-1}}<\infty .
$$

So, the assertion $u_{\star} \in Q_{0<\alpha<2^{-1}}\left(\mathbb{R}^{n}\right)$ follows from Lemma 2.4. 
Lemma 2.6. For $n-1 \in \mathbb{N}$ let $\mathbf{a}=\left(a_{1}, \ldots, a_{n}\right)$ be with $0<a_{1} \leq a_{2} \leq \ldots \leq a_{n}=1$. Given $r>0$ set

$$
\left\{\begin{array}{l}
\left(u_{\star}\right)_{r}(x)=u_{\star}\left(r^{-1} x\right) \\
P_{\mathbf{a}, r}=\left\{x=\left(x_{1}, \ldots, x_{n}\right) \in \mathbb{R}^{n}:\left|x_{1}\right| \leq a_{1} r, \ldots,\left|x_{n}\right| \leq a_{n} r\right\} \\
\left(u_{\star}\right)_{\mathbf{a}, r}=\frac{\left(u_{\star}\right)_{r} \chi_{P_{\mathbf{a}, r}}}{\left|P_{\mathbf{a}, r}\right|}=\frac{\left(u_{\star}\right)_{r} \chi_{P_{\mathbf{a}, r}}}{(2 r)^{n} a_{1} \ldots a_{n}} \\
c_{\mathbf{a}}=\int_{\mathbb{R}^{n}}\left|\left(u_{\star}\right)_{\mathbf{a}, r}(x)\right| d x=f_{P_{\mathbf{a}, r}}\left|\left(u_{\star}\right)_{r}(x)\right| d x=f_{P_{\mathbf{a}, 1}}\left|u_{\star}(x)\right| d x .
\end{array}\right.
$$

If $h \in L^{1}\left(\mathbb{R}^{n}\right)$, then there exists a subsequence $\left\{r_{j}\right\}$ converging to 0 such that for any rational point $\mathbf{a} \in \mathbb{R}^{n}$ one has that

$$
\left\{\begin{array}{l}
\left(u_{\star}\right)_{\mathbf{a}, r_{j}} * h(y)=\int_{\mathbb{R}^{n}}\left(u_{\star}\right)_{\mathbf{a}, r_{j}}(z) h(y-z) d z \rightarrow 0 ; \\
\left|\left(u_{\star}\right)_{\mathbf{a}, r_{j}}\right| * h(y)=\int_{\mathbb{R}^{n}}\left|\left(u_{\star}\right)_{\mathbf{a}, r_{j}}(z)\right| h(y-z) d z \rightarrow c_{\mathbf{a}} h(y),
\end{array}\right.
$$

holds for almost all $y \in \mathbb{R}^{n}$.

Proof. The argument is similar to the proof of [9, Lemma 8].

Proof of Theorem 1.2. We are about to use Reimann's procedure in [9]. Rather than showing that $f$ is quasiconformal, we prove that $f^{-1}$ (the inverse of $f$ ) is quasiconformal. It suffices to verify that

$$
\sup _{y \in \partial B(0,1)}\left|\left(D f^{-1}(x)\right) y\right|^{n} \lesssim J_{f^{-1}}(x)
$$

holds for almost all $x \in \mathbb{R}^{n}$ where $D f^{-1}$ and $J_{f^{-1}}$ are the formal derivative and Jacobian determinant of $f^{-1}$ (cf. [4, p.250]). Since $f^{-1}$ is absolutely continuous with respect to the $n$-dimensional Lebesgue measure, one has

$$
J_{f-1}(x)=\lim _{r \rightarrow 0} \frac{\left|f^{-1}(B(x, r))\right|}{|B(x, r)|}
$$

almost everywhere and $J_{f^{-1}} \in L_{\text {loc }}^{1}\left(\mathbb{R}^{n}\right)$ where the absolute values right after $\lim _{r \rightarrow 0}$ stand for the $n$-dimensional Lebesgue measures of the sets $f^{-1}(B(x, r))$ and $B(x, r)$ respectively. Also our hypothesis implies that $f^{-1}$ is (totally) differentiable almost everywhere, and $J_{f^{-1}}>0$ holds almost everywhere. We may assume $J_{f^{-1}}(0)>0$ and $h=\chi_{B(0,1)} J_{f^{-1}}$ in Lemma 2.6. Up to some rotation, translation and scaling which preserve the $Q_{\alpha}\left(\mathbb{R}^{n}\right)$-norm, we may also assume

$$
\left\{\begin{array}{l}
f^{-1}(0)=0 \\
D f^{-1}(0)=\operatorname{diag}\left\{\lambda_{1}, \ldots, \lambda_{n}\right\} \\
\lambda_{1} \geq \ldots \geq \lambda_{n}=1
\end{array}\right.
$$


and so are required to verify

$$
(\sharp) \quad \lambda_{1}^{n} \lesssim \lambda_{1} \ldots \lambda_{n} .
$$

Given any sufficiently small $\varepsilon>0$, we choose

$$
\mathbf{a}_{m}=\left(a_{m 1}, \ldots, a_{m n}\right)
$$

rationally such that

$$
0<a_{m 1} \leq a_{m 2} \leq \ldots \leq a_{m n}=1 \quad \& \sum_{k=1}^{n}\left|a_{m k} \lambda_{k}-1\right|<\varepsilon .
$$

Let

$$
\left\{\begin{array}{l}
P_{r}=\left\{z=\left(z_{1}, \ldots, z_{n}\right) \in \mathbb{R}^{n}:\left|z_{1}\right|, \ldots,\left|z_{n}\right| \leq r\right\} ; \\
P_{\mathbf{a}_{m}, r}=\left\{z=\left(z_{1}, \ldots, z_{n}\right) \in \mathbb{R}^{n}:\left|z_{1}\right| \leq a_{m 1} r, \ldots,\left|z_{n}\right| \leq a_{m n} r\right\} .
\end{array}\right.
$$

Upon using Lemma 2.6 with $\mathbf{a}=\mathbf{a}_{m}$, we write

$$
c_{\mathbf{a}_{m}}=f_{P_{\mathbf{a}_{m}, 1}}\left|u_{\star}(x)\right| d x .
$$

By the definition of $u_{\star}$ as in Corollary 2.5 with $\mathbf{a}=\mathbf{a}_{m}$ we have

$$
\text { (†) } \quad c_{\mathbf{a}_{m}} \gtrsim-\ln a_{m 1} \text {. }
$$

Indeed, if $n=2$, then

$$
0<a_{m 1} \leq 1=a_{m 2}
$$

derives

$$
\begin{aligned}
f_{P_{\mathbf{a}_{m}, 1}}\left|u_{\star}(x)\right| d x & =\left(4 a_{m 1} a_{m 2}\right)^{-1} \int_{-a_{m 1}}^{a_{m 1}} \int_{-a_{m 2}}^{a_{m 2}} \max \left\{0, \ln \left(x_{1}^{-2}\right)\right\}\left|\phi\left(x_{2}\right)\right| d x_{1} d x_{2} \\
& \gtrsim\left(a_{m 1} a_{m 2}\right)^{-1} \int_{0}^{a_{m 2}}\left(\int_{0}^{a_{m 1}} \ln \left(x_{1}^{-2}\right) d x_{1}\right) x_{2} d x_{2} \\
& \gtrsim-\ln a_{m 1} .
\end{aligned}
$$

Furthermore, if $n \geq 3$, then a similar argument, along with

$$
\psi(t)=1 \quad \forall|t| \leq 1
$$

will also ensure $(\dagger)$.

In this way, for a sufficiently small $r<\delta_{1}$ we have that $f^{-1}\left(P_{\mathbf{a}_{m}, r}\right)$ contains

$$
R=\left\{z=\left(z_{1}, \ldots, z_{n}\right) \in \mathbb{R}^{n}:\left|z_{1}\right|, \ldots,\left|z_{n}\right| \leq r(1-\varepsilon)\right\}
$$


and is contained in

$$
S=\left\{z=\left(z_{1}, \ldots, z_{n}\right) \in \mathbb{R}^{n}:\left|z_{1}\right|, \ldots,\left|z_{n}\right| \leq r(1+\varepsilon)\right\}
$$

In fact, this can be obtained by the differentiability of $f^{-1} \& f$ at 0 , and

$$
D f^{-1}(0)=\operatorname{diag}\left\{\lambda_{1}, \ldots, \lambda_{n}\right\} \quad \& \quad D f(0)=\operatorname{diag}\left\{\lambda_{1}^{-1}, \ldots, \lambda_{n}^{-1}\right\} .
$$

By virtue of the assumption on $f$ and the function $u_{\star}$ constructed in Corollary 2.5 , we have

$$
\begin{aligned}
(\ddagger)\left\|\mathbf{C}_{f} u_{\star}\right\|_{Q_{-\frac{n}{2}}\left(\mathbb{R}^{n}\right)} & \lesssim\left\|\mathbf{C}_{f} u_{\star}\right\|_{Q_{0<\alpha<2^{-1}}\left(\mathbb{R}^{n}\right)} \lesssim\left\|\left(u_{\star}\right)_{r}\right\|_{Q_{0<\alpha<2^{-1}}\left(\mathbb{R}^{n}\right)} \\
& \lesssim\left\|u_{\star}\right\|_{Q_{0<\alpha<2^{-1}}\left(\mathbb{R}^{n}\right)} \lesssim 1 .
\end{aligned}
$$

Since

$$
Q_{0<\alpha<2^{-1}}\left(\mathbb{R}^{n}\right) \subset B M O\left(\mathbb{R}^{n}\right)=Q_{-\frac{n}{2}}\left(\mathbb{R}^{n}\right)
$$

we are required to control

$$
\left\|\mathbf{C}_{f} u_{\star}\right\|_{B M O\left(\mathbb{R}^{n}\right)}=\left\|\mathbf{C}_{f} u_{\star}\right\|_{Q_{-\frac{n}{2}}\left(\mathbb{R}^{n}\right)}
$$

via

$$
\left\|\mathbf{C}_{f} u_{\star}\right\|_{B M O\left(\mathbb{R}^{n}\right)} \gtrsim f_{f^{-1}\left(P_{\mathbf{a}_{m}, r}\right)}\left|\mathbf{C}_{f} u_{\star}(x)-f_{f^{-1}\left(P_{\mathbf{a}_{m}, r}\right)} \mathbf{C}_{f} u_{\star}(y) d y\right| d x .
$$

Note that if

$$
h(x)= \begin{cases}J_{f}(x) & \text { for } x \in B(0,1) \\ 0 & \text { for } x \in \mathbb{R}^{n} \backslash B(0,1)\end{cases}
$$

then

$$
\begin{aligned}
f_{f^{-1}\left(P_{\mathbf{a}_{m}, r}\right)} \mathbf{C}_{f} u_{\star}(x) d x & =\frac{\left|P_{\mathbf{a}_{m}, r}\right|}{\left|f^{-1}\left(P_{\mathbf{a}_{m}, r}\right)\right|} f_{P_{\mathbf{a}_{m}, r}}\left(u_{\star}\right)_{r}(z) J_{f^{-1}}(z) d z \\
& =\frac{\left|P_{\mathbf{a}_{m}, r}\right|}{\left|f^{-1}\left(P_{\mathbf{a}_{m}, r}\right)\right|}\left(u_{\star}\right)_{\mathbf{a}_{m}, r} * h(0) .
\end{aligned}
$$

So, upon applying Lemma 2.6, we obtain a constant $\delta_{2} \in\left(0, \delta_{1}\right)$ and a sequence $r_{j}<\delta_{2}$ such that

$$
\left|f_{f^{-1}\left(P_{\mathbf{a}_{m}, r}\right)}\left(\mathbf{C}_{f}\left(u_{\star}\right)_{r_{j}}\right)(x) d x\right| \leq \varepsilon \quad \forall \quad \mathbf{a}_{m}
$$


Accordingly,

$$
\left\|\mathbf{C}_{f} u_{\star}\right\|_{B M O\left(\mathbb{R}^{n}\right)} \geq f_{f^{-1}\left(P_{\mathbf{a}_{m}, r}\right)}\left|\mathbf{C}_{f} u_{\star}(x)\right| d x-\varepsilon \quad \forall \quad r \in(0, \infty) .
$$

Similarly, we have

$$
\begin{aligned}
f_{f^{-1}\left(P_{\mathbf{a}_{m}, r}\right)}\left|\mathbf{C}_{f} u_{\star}(x)\right| d x & =\left(\frac{\left|P_{\mathbf{a}_{m}, r}\right|}{\mid f^{-1}\left(P_{\mathbf{a}_{m}, r}\right)}\right) f_{P_{\mathbf{a}_{m}, r}}\left|\left(u_{\star}\right)_{r}(z)\right| J_{f^{-1}}(z) d z \\
& =\left(\frac{\left|P_{\mathbf{a}_{m}, r}\right|}{\mid f^{-1}\left(P_{\mathbf{a}_{m}, r}\right)}\right)\left|\left(u_{\star}\right)_{\mathbf{a}_{m}, r}\right| * h(0),
\end{aligned}
$$

thereby using Lemma 2.6 to discover

$$
\liminf _{r_{j} \rightarrow 0} f_{f^{-1}\left(P_{\mathbf{a}_{m}, r_{j}}\right)}\left|\left(\mathbf{C}_{f}\left(u_{\star}\right)_{\mathbf{a}_{m}, r_{j}}\right)(x)\right| d x=\left(\liminf _{r_{j} \rightarrow 0} \frac{\left|P_{\mathbf{a}_{m}, r_{j}}\right|}{\mid f^{-1}\left(P_{\mathbf{a}_{m}, r_{j}}\right)}\right) c_{\mathbf{a}_{m}} h(0) .
$$

For $r_{j}<\delta_{1}$, we utilize

$$
1-\varepsilon \leq a_{m k} \lambda_{k} \leq 1+\varepsilon \quad \forall \quad k \in\{1, \ldots, n\},
$$

to deduce

$$
\left(\frac{\left|P_{\mathbf{a}_{m}, r_{j}}\right|}{\left|f^{-1}\left(P_{\mathbf{a}_{m}, r_{j}}\right)\right|}\right) h(0) \geq(1+\varepsilon)^{-n}\left(a_{m 1} \ldots a_{m n}\right)\left(\lambda_{1} \ldots \lambda_{n}\right) \geq\left(\frac{1-\varepsilon}{1+\varepsilon}\right)^{n},
$$

whence

$$
\liminf _{r_{j} \rightarrow 0} f_{f^{-1}\left(P_{\mathbf{a}, r}\right)}\left|\left(\mathbf{C}_{f}\left(u_{\star}\right)_{r_{j}}\right)(x)\right| d x \geq\left(\frac{1-\varepsilon}{1+\varepsilon}\right)^{n} c_{\mathbf{a}_{m}},
$$

which in turn implies

$$
\left\|\mathbf{C}_{f} u_{\star}\right\|_{B M O\left(\mathbb{R}^{n}\right)} \geq\left(\frac{1-\varepsilon}{1+\varepsilon}\right)^{n} c_{\mathbf{a}_{m}}-\varepsilon .
$$

Upon combining this with $(\dagger)-(\ddagger)$, we achieve a constant $\varkappa>0$ (independent of $\mathbf{a}_{m}$ ) such that

$$
-\ln a_{m 1} \leq \varkappa \quad \& \quad a_{m 1} \geq e^{-\varkappa}
$$

Consequently, we gain

$$
1=\lambda_{n} \leq \lambda_{n-1} \leq \ldots \leq \lambda_{1} \leq 2 e^{\varkappa}
$$

thereby reaching $(\sharp)$. 


\section{Validation of Theorem 1.5}

In order to prove Theorem 1.5, we need the concept of a $Q_{\alpha}\left(\mathbb{R}^{n}\right)$-capacity. For $(\alpha, n) \in(-\infty, 1) \times \mathbb{N}$ and any pair of disjoint continua $E, F \subset \mathbb{R}^{n}$, let

$$
\operatorname{Cap}_{Q_{\alpha}\left(\mathbb{R}^{n}\right)}(E, F)=\inf \left\{\|u\|_{Q_{\alpha}\left(\mathbb{R}^{n}\right)}^{2}: u \in \Delta_{\alpha}(E, F)\right\}
$$

be the $Q_{\alpha}\left(\mathbb{R}^{n}\right)$-capacity of the pair $(E, F)$, where $\Delta_{\alpha}(E, F)$ is the class of all continuous functions $u \in Q_{\alpha}\left(\mathbb{R}^{n}\right)$ enjoying

$$
\begin{cases}0 \leq u \leq 1 & \text { on } \mathbb{R}^{n} \\ u=0 & \text { on } E ; \\ u=1 & \text { on } F\end{cases}
$$

Obviously, if $\widetilde{E} \& \widetilde{F}$ are disjoint continua satisfying $E \subseteq \widetilde{E} \& F \subseteq \widetilde{F}$, then

$$
\operatorname{Cap}_{Q_{\alpha}\left(\mathbb{R}^{n}\right)}(E, F) \leq \operatorname{Cap}_{Q_{\alpha}\left(\mathbb{R}^{n}\right)}(\widetilde{E}, \widetilde{F}) .
$$

Moreover, we have

Lemma 3.1. Given a constant $\delta \in(0, \infty)$ let $n=1 \& \alpha \in\left(0,2^{-1}\right]$ or $n=2 \& \alpha \in$ $\left(2^{-1}, 1\right)$. If $E \& F$ are disjoint continua in $\mathbb{R}^{n}$ such that their diameters diam $E \&$ $\operatorname{diam} F$ and Euclidean distance $\operatorname{dist}(E, F)$ obey

$$
\min \{\operatorname{diam} E, \operatorname{diam} F\} \geq \delta \operatorname{dist}(E, F)>0,
$$

then

$$
\operatorname{Cap}_{Q_{\alpha}\left(\mathbb{R}^{n}\right)}(E, F) \gtrsim 1
$$

Proof. Without loss of generality we may assume

$$
\operatorname{diam} E=\operatorname{diam} F \geq \delta \operatorname{dist}(E, F) .
$$

If

$$
x_{0} \in E \quad \& \quad r=\left(2+\delta^{-1}\right) \operatorname{diam} E
$$

then

$$
E, F \subseteq B\left(x_{0}, r\right) .
$$

Thanks to either $n=1 \& \alpha \in\left(0,2^{-1}\right]$ or $n=2 \& \alpha \in\left(2^{-1}, 1\right)$, we may assume

$$
\left\{\begin{array}{l}
u \in \Delta_{\alpha}(E, F) \\
u_{B\left(x_{0}, r\right) \geq 2^{-1}} \\
0<\varepsilon \leq 1-n+2 \alpha .
\end{array}\right.
$$


For every $x \in E$ and $\rho>0$ we utilize

$$
\begin{aligned}
\Phi_{\alpha}(u, B(x, \rho)) & =\rho^{2 \alpha-n} \int_{B(x, \rho)} \int_{B(x, \rho)} \frac{|u(z)-u(w)|^{2}}{|z-w|^{n+2 \alpha}} d z d w \\
& \gtrsim f_{B(x, \rho)} f_{B(x, \rho)}|u(z)-u(w)| d z d w
\end{aligned}
$$

to estimate

$$
\begin{aligned}
2^{-1} & \leq\left|u(x)-u_{B\left(x_{0}, r\right)}\right| \\
& \leq \sum_{i=-1}^{\infty}\left|u_{B\left(x, 2^{-i} r\right)}-u_{B\left(x, 2^{-i-1} r\right)}\right|+\left|u_{B(x, 2 r)}-u_{B\left(x_{0}, r\right)}\right| \\
& \lesssim \sum_{i=-1}^{\infty}\left(f_{B\left(x, 2^{-i} r\right)} f_{B\left(x, 2^{-i} r\right)}|u(z)-u(w)|^{2} d z d w\right)^{2^{-1}} \\
& \lesssim \sum_{i=-1}^{\infty}\left(\Phi_{\alpha}\left(u, B\left(x, 2^{-i} r\right)\right)\right)^{2^{-1}} \\
& \lesssim \sum_{i=-1}^{\infty}\left(2^{-i} r\right)^{\frac{\varepsilon}{2}} \sup _{t \leq 2 r} t^{-\frac{\varepsilon}{2}}\left[\Phi_{\alpha}(u, B(x, t))\right]^{2^{-1}} \\
& \lesssim r^{\frac{\varepsilon}{2}} \sup _{t \leq 2 r} t^{-\frac{\varepsilon}{2}}\left(\Phi_{\alpha}(u, B(x, t))\right)^{2^{-1}} .
\end{aligned}
$$

Accordingly, for each $x \in E$ there exists a $t_{x} \in(0,2 r]$ such that

$$
\left\{\begin{array}{l}
1 \lesssim r^{\varepsilon} t_{x}^{-\varepsilon} \Phi_{\alpha}\left(u, B\left(x, t_{x}\right)\right) \\
t_{x}^{n-2 \alpha+\varepsilon} \lesssim r^{\varepsilon} \int_{B\left(x, t_{x}\right)} \int_{B\left(x, t_{x}\right)} \frac{|u(z)-u(w)|^{2}}{|z-w|^{n+2 \alpha}} d z d w .
\end{array}\right.
$$

By the Vitali covering lemma, we can find points $x_{i} \in E$ and radii $r_{i}>0$ as above such that ball $B\left(x_{i}, t_{i}\right)$ are mutually disjoint and $E \subseteq \bigcup_{i} B\left(x_{i}, 5 t_{i}\right)$. Hence,

$$
\operatorname{diam} E \lesssim \sum_{i=-1}^{\infty} t_{i} \lesssim r^{\frac{\varepsilon}{n-2 \alpha+\varepsilon}} \sum_{i=-1}^{\infty}\left(\int_{B\left(x_{i}, t_{i}\right)} \int_{B\left(x_{i}, t_{i}\right)} \frac{|u(z)-u(w)|^{2}}{|z-w|^{n+2 \alpha}} d z d w\right)^{\frac{1}{n-2 \alpha+\varepsilon}}
$$

Upon noticing $1 /(n-2 \alpha+\varepsilon) \geq 1$, we obtain

$$
\begin{aligned}
\frac{r}{2+\delta^{-1}} & \lesssim r^{\frac{\varepsilon}{n-2 \alpha+\varepsilon}}\left(\sum_{i=-1}^{\infty} \int_{B\left(x_{i}, t_{i}\right)} \int_{B\left(x_{i}, t_{i}\right)} \frac{|u(z)-u(w)|^{2}}{|z-w|^{n+2 \alpha}} d z d w\right)^{\frac{1}{n-2 \alpha+\varepsilon}} \\
& \lesssim r^{\frac{\varepsilon}{n-2 \alpha+\varepsilon}}\left(\int_{B\left(x_{0}, 4 r\right)} \int_{B\left(x_{0}, 4 r\right)} \frac{|u(z)-u(w)|^{2}}{|z-w|^{n+2 \alpha}} d z d w\right)^{\frac{1}{n-2 \alpha+\varepsilon}}
\end{aligned}
$$


whence

$$
\Phi_{\alpha}\left(u, B\left(x_{0}, 4 r\right)\right) \gtrsim 1
$$

which yields

$$
\operatorname{Cap}_{Q_{\alpha}\left(\mathbb{R}^{n}\right)}(E, F) \gtrsim 1 \text {. }
$$

Proof of Theorem 1.5. By the metric characterization of a quasiconformal mapping (cf. [7]), it is enough to validate that if

$$
\left\{\begin{array}{l}
\ell(f, r)=\inf \left\{\left|f(x)-f\left(x_{0}\right)\right|:\left|x-x_{0}\right| \geq r\right\} \\
L(f, r)=\sup \left\{\left|f(x)-f\left(x_{0}\right)\right|:\left|x-x_{0}\right| \leq r\right\} \\
\left(x_{0}, r\right) \in \mathbb{R}^{2} \times(0, \infty)
\end{array}\right.
$$

then

$$
L(f, r) \leq c(f) \ell(f, r),
$$

where $c(f)$ is a positive constant depending on $f$.

To this end, if

$$
v(y)= \begin{cases}1 & \text { as }\left|y-x_{0}\right| \leq \ell(f, r) \\ \frac{\ln L(f, r)-\ln \left|y-x_{0}\right|}{\ln L(f, r)-\ln \ell(f, r)} & \text { as } \ell(f, r) \leq\left|y-x_{0}\right| \leq L(f, r) \\ 0 & \text { as }\left|y-x_{0}\right| \geq L(f, r)\end{cases}
$$

then

$$
|\nabla v(y)|= \begin{cases}0 & \text { as }\left|y-x_{0}\right| \leq \ell(f, r) \\ \frac{\left|y-x_{0}\right|^{-1}}{\ln L(f, r)-\ln \ell(f, r)} & \text { as } \ell(f, r) \leq\left|y-x_{0}\right| \leq L(f, r) \\ 0 & \text { as }\left|y-x_{0}\right| \geq L(f, r)\end{cases}
$$

and hence

$$
\begin{aligned}
\|v\|_{W^{1,2}\left(\mathbb{R}^{2}\right)}^{2} & =\int_{\mathbb{R}^{2}}|\nabla v(y)|^{2} d y \\
& =\left(\ln \frac{L(f, r)}{\ell(f, r)}\right)^{-2} \int_{l \leq\left|y-x_{0}\right| \leq L} \frac{d y}{\left|y-x_{0}\right|^{2}} \\
& \lesssim\left(\ln \frac{L(f, r)}{\ell(f, r)}\right)^{-1} .
\end{aligned}
$$

This last estimation, along with [10, Theorem 4.1] under $n=2 \& \alpha<1$, implies

$$
\|v\|_{Q_{2-1}<\alpha<1}\left(\mathbb{R}^{2}\right) \lesssim\|v\|_{W^{1,2}\left(\mathbb{R}^{2}\right)} \lesssim\left(\ln \frac{L(f, r)}{\ell(f, r)}\right)^{-2^{-1}} .
$$


Let

$$
E=f^{-1}\left(B\left(f\left(x_{0}\right), \ell\right)\right)
$$

i.e., the preimage of $B\left(f\left(x_{0}\right), \ell\right)$ under $f$. Then $E$ is connected and enjoys

$$
E \subseteq B\left(x_{0}, r\right) \quad \& \quad \operatorname{diam} E \geq r
$$

Moreover, observe that as the connected preimage of $\mathbb{R}^{2} \backslash B\left(f\left(x_{0}\right), L\right)$ under $f$,

$$
f^{-1}\left(\mathbb{R}^{2} \backslash B\left(f\left(x_{0}\right), L\right)\right)
$$

joins

$$
\bar{B}\left(x_{0}, r\right)=\left\{x \in \mathbb{R}^{2}:\left|x-x_{0}\right| \leq r\right\} \quad \& \quad \mathbb{R}^{2} \backslash B\left(x_{0}, 2 r\right) .
$$

So we can find a connected continum $F$ such that it is contained in

$$
f^{-1}\left(\mathbb{R}^{2} \backslash B\left(f\left(x_{0}\right), L\right)\right)
$$

and joins $\bar{B}\left(x_{0}, r\right)$ and $\mathbb{R}^{2} \backslash B\left(x_{0}, 2 r\right)$, and consequently we may assume

$$
F \subseteq \bar{B}\left(x_{0}, 2 r\right) \backslash B\left(x_{0}, r\right)
$$

Obviously, we have

$$
\operatorname{diam} F \geq r \quad \& \quad 0<\operatorname{dist}(E, F) \leq 5 r \leq 10 \min \{\operatorname{diam} E, \operatorname{diam} F\} .
$$

Upon applying Lemma 3.1 under $n=2 \& 2^{-1}<\alpha<1$ we discover

$$
\operatorname{Cap}_{Q_{\alpha}\left(\mathbb{R}^{2}\right)}(E, F) \gtrsim 1
$$

thereby arriving at the required inequality

$$
\ln \frac{L(f, r)}{\ell(f, r)} \lesssim 1
$$

Acknowledgement. We would like to thank the referee and P. Koskela for their useful and constructive comments on the paper, as well as Y. Zhang for his example showing that if $n=2$ and $0<\alpha<2^{-1}$ (cf. Theorem 1.2) then there are two parallel sets $E, F \subset \mathbb{R}^{2}$ such that the last capacity estimation fails. 


\section{References}

1. Astala, K., A remark on quasi-conformal mappings and BMO-functions, Michigan Math. J. 30 (1983), 209-212. MR0718266

2. Essén, M., Janson, S., Peng, L. and XiAo, J., $Q$ spaces of several real variables, Indiana Univ. Math. J. 49 (2000), 575-615. MR1793683

3. Gотон, Y., On composition operators which preserve BMO, Pacific J. Math. 201 (2001), 289-307. MR1875895

4. Heinonen, J., Kilpeläinen, T. and Martio, O., Nonlinear Potential Theory of Degenerate Elliptic Equations, Dover, New York, 2006. MR2305115

5. John, F. and Nirenberg, L., On functions of bounded mean oscillation, Comm. Pure Appl. Math. 18 (1965), 415-426. MR0131498

6. Koch, H., Koskela, P., Saksman, E. and Soto, T., Bounded compositions on scaling invariant Besov spaces, J. Funct. Anal. 266 (2014), 2765-2788. MR3158708

7. Koskela, P., Lectures on Quasiconformal and Quasisymmetrical Mappings, University of Jyväskylä, 2011.

8. Koskela, P., Xiao, J., Zhang, Y. and Zhou, Y., A quasiconformal composition problem for the $Q$-spaces, J. Eur. Math. Soc. (JEMS) 19 (2017), 11591187. MR3626553

9. Reimann, H. M., Functions of bounded mean oscillation and quasiconformal mappings, Comment. Math. Helv. 49 (1974), 260-276. MR0361067

10. XIAO, J., A sharp Sobolev trace inequality for the fractional-order derivatives, Bull. Sci. Math. 130 (2006), 87-96. MR2197182

Jie Xiao

Department of Mathematics and Statistics Memorial University

St. John's NL A1C 5S7

Canada

jxiao@mun.ca
Yuan Zhou

Department of Mathematics

Beihang University

Beijing 100191

China

yuanzhou@buaa.edu.cn

Received March 6, 2018

in revised form April 4, 2019 